\title{
Characterization of Biological Material Adsorption to the Surface of Nanoparticles without a Prior Separation Step: a Case Study of Glioblastoma-Targeting Peptide and Lipid Nanocapsules
}

\author{
Claire Gazaille' - Marion Sicot ' Marthe Akiki ${ }^{\prime}$ - Nolwenn Lautram ' - Aurélien Dupont ${ }^{2}$. \\ Patrick Saulnier' • Joël Eyer ' • Guillaume Bastiat ' (D) \\ Received: 12 February 2021 / Accepted: 23 March 2021 / Published online: 7 April 2021 \\ (C) The Author(s), under exclusive licence to Springer Science+Business Media, LLC, part of Springer Nature 2021
}

\begin{abstract}
Purpose Current preclinical therapeutic strategies involving nanomedicine require increasingly sophisticated nanosystems and the characterization of the complexity of such nanoassemblies is becoming a major issue. Accurate characterization is often the factor that can accelerate the translational approaches of nanomedicines and their pharmaceutical development to reach the clinic faster. We conducted a case study involving the adsorption of the NFL-TBS.40-63 (NFL) peptide (derived from neurofilaments) to the surface of lipid nanocapsules (LNCs) (a combined nanosystem used to target glioblastoma cells) to develop an analytical approach combining the separation and the quantification in a single step, leading to the characterization of the proportion of free peptide and thus the proportion of peptide adsorbed to the lipid nanocapsule surface.
\end{abstract}

Methods LNG suspensions, NFL peptide solution and LNC/ NFL peptide mixtures were characterized using a SizeExclusion Chromatography method (with a chromatographic apparatus). In addition, this method was compared to centrifugal-filtration devices, currently used in literature for this case study.

Results Combining the steps for separation and characterization in one single sequence improved the accuracy and robustness of the data and led to reproducible results. Moreover the data deviation observed for the centrifugal-filtration devices demonstrated the limits for this increasingly used characterization approach, explained by the poor separation quality and highlighting the importance for the method optimization.

Guillaume Bastiat

guillaume.bastiat@univ-angers.fr

University of Angers, Inserm, CNRS, MINT, SFR ICAT,

F-49000 Angers, France

2 University of Rennes, CNRS, Inserm, BIOSIT, UMS 3480, US_S 018 , F-35000 Rennes, France
The high potential of the technique was shown, proving that H-bond and/or electrostatic interactions mediate adsorption of the NFL peptide to the surface of LNCs.

Conclusions Used only as a characterization tool, the process using chromatographic apparatus is less time and solvent consuming than classical Size-Exclusion Chromatography columns only used for separation. It could be a promising tool for the scientific community for characterizing the interactions of other combinations of nanosystems and active biological agents.

KEY WORDS adsorption · centrifugal-filtration devices · lipid nanocapsules $\cdot$ peptide $\cdot$ size-exclusion chromatography

\section{INTRODUCTION}

The in vitro and in vivo physicochemical characterization of nanomaterials, such as nanomedicines, is becoming a major issue due to the increasing complexity of the developed nanosystems. Indeed, preclinical therapeutic strategies using nanomedicines focus on stimuli-responsive nanosystems, specific targeting, or both, while ensuring an optimal drugencapsulation rate inside the nanosystems (1-7). The classical methods used to characterize nanomedicines may be inappropriate, leading to erroneous results when used to characterize the increasingly complex nanosystems currently under study. In terms of clinical perspectives, appropriate characterization facilitates larger-scale development of nanomedicines and their potential industrial production (8). The translation of promising drug-delivery systems may require the adaptation of existing methods to ensure their quality, efficacy, and safety.

Increasing attention is being given to analytical methods in project calls and several academic laboratories have developed true expertise in the characterization of nanosystems. Similar to the Nanotechnology Characterization Laboratory 
(NCL), funded in 2004 under the leadership of the US Food and Drug Administration (FDA) and the National Institute of Standards and Technology (NIST), the European Nanomedicine Characterization Laboratory (involving nine partners) was created in 2015, funded by the European Union's Horizon 2020 Research and Innovation Program. These institutes help the scientific community in developing harmonized analytical protocols for nanomedicine characterization. Moreover, a recent publication reported suitable existing standard methods for the evaluation of nanomedicines (9).

Among the methods used to separate nanoparticles from drugs or biological materials is centrifugal-filtration. Not only used in biological research, these devices are often used to purify and/or separate nanoparticles from smaller molecules (biological materials, drugs, etc.) prior to their characterization, mainly due to their speed and ease of implementation (10-13). More often than not, published studies reporting the use of this method do not show the controls used, raising doubts about the quality of the separation. Indeed, the selectivity of centrifugal filters is not as precise as may be expected: biological material can cross the filters, even if their molecular weight is higher than the molecular cut-off. In addition, they can be trapped on the device itself, even if their molecular weight is lower than the molecular cut-off $(14,15)$. Such nonselectivity may have an impact on the quality of nanosystem/ biological molecule separations, perhaps leading to inaccurate results.

Our group has studied a new therapeutic strategy against glioblastoma, using lipid nanocapsules (LNCs) that can specifically target glioblastoma cells. NFL-TBS.40-63 (NFL), a peptide corresponding to the sequence of a tubulin-binding site on neurofilaments, can specifically target glioblastoma cells without affecting normal brain cells (16-18). This peptide can be adsorbed to the surface of LNGs as a recognition ligand against glioblastoma cells. This association has shown significant in vitro and in vivo efficacy against glioblastoma cells using drug-loaded LNCs relative to drug-loaded LNCs without NFL peptide $(13,19,20)$. For this application, it is important to quantify the amount of NFL peptide adsorbed to the surface of the LNCs to adjust the proportion to optimize specific targeting.

Here, we used LNCs and the NFL peptide as models for a case study to develop a method to separate and characterize the LNC/NFL peptide mixtures in a single step. The method is based on the Size-Exclusion Chromatography (SEG) technique, using an Ultra-Performance Liquid-Chromatography (UPLG) apparatus. It is the first time to our knowledge that LNC suspensions and more broadly nanoparticle suspensions were injected in such SEC columns. The same samples were tested to challenge the current protocol: centrifugal-filtration device for the separation and BCA assays for the free NFL quantification. Only this technique was exclusively used to quantify the adsorption of NFL peptide at the surface of LNGs in previous work $(13,20)$, and no additional characterization was done to confirm the data. Using the SEC/UPLC technique, no additional purification process was needed to separate the nanosystems and non-adsorbed biological material before their characterization, avoiding the bias that could be encountered. Regarding the protocol described in literature, it seems that the centrifugal-filtration devices are not perfectly adapted to the separation of LNGs and free NFL peptide, leading to questionable quantification. Indeed, we identified certain limitations, such as i) LNCs alone can pass through the filter, despite their having a molecular weight above the molecular cut-off of the filter, ii) the NFL peptide alone is partially retained in the filter, despite having a molecular weight below the molecular cutoff of the filter, and iii) the separation of the LNC/NFL peptide mixture does not lead to reproducible results. These limitations were established by assessing the separation of LNGs alone, the NFL peptide alone, and LNC/NFL peptide mixtures after incubation. Finally, using the SEC/UPLC technique, we characterized the parameters that can influence the adsorption of the NFL peptide to the LNC surface, i.e. the size and surface properties of the LNCs, showing great perspectives for this characterization system.

\section{MATERIALS AND METHODS}

\section{Materials}

Span ${ }^{\circledR} 80$ (sorbitane monooleate) (Span), sodium cholesteryl sulfate (SChol), and didodecyldimethylammonium bromide (DDAB) were supplied by Sigma-Aldrich (Saint-QuentinFallavier, France). Kolliphor® HS15 (mixture of free polyethylene glycol 660 and polyethylene glycol 660 hydroxystearate) (Kol) was purchased from BASF (Ludwigshafen, Germany). Labrafac ${ }^{\circledR}$ WL 1349 (caprylic-capric acid triglycerides) (Lab) was provided by Gattefossé S.A. (Saint-Priest, France). $100 \mathrm{~K}$ Amicon ${ }^{\circledR}$ Ultra filters (filter cut-off $=100 \mathrm{kDa}$ ) were supplied by Merck (Darmstadt, Allemagne). NaCl was purchased from Prolabo (Fontenay-sous-bois, France). Deionized water was obtained from a Milli-Q plus ${ }^{\circledR}$ system (Millipore, Bilerica, USA). The biotinylated NFL-TBS.40-63 peptide (NFL) was synthesized by the PolyPeptide group (Strasbourg, France).

\section{LNC Formulation}

The LNG formulation process was based on an already reported and patented phase-inversion method $(21,22)$. The quantities of $\mathrm{NaCl}$, Kol, Span, Lab, DDAB, SChol, and water were precisely weighed for each formulation (Supplementary Material Table 1). Three temperature cycles, from 50 to $85^{\circ} \mathrm{C}$, were performed under magnetic stirring and the 
emulsified system was modified from an oil-in-water emulsion at low temperature to a water-in-oil emulsion at high temperature. During the last cooling phase, deionized water $\left(\right.$ at $4^{\circ} \mathrm{C}$ ) was quickly added at the temperature of the phase inversion zone, approximately $65^{\circ} \mathrm{C}$, always under magnetic stirring, resulting in the LNG suspension. Then, the LNC suspensions were cooled under magnetic stirring for 5 min until reaching room temperature. The final LNC concentrations, LNC total surface area, and proportions of Span, SChol, and DDAB in the LNCs were calculated based on the sizes of the LNCs and the volume of deionized water added at the end of the formulation process (Supplementary Material - Supplementary discussion) and are reported in Supplementary Material Table 2.

\section{LNC Characterization}

The zeta potential ( $\mathrm{ZP})$ and the size distribution, i.e. Zaverage diameter (Z-ave) and polydispersity index $(\mathrm{PdI})$, of LNCs in suspension were measured using a Zetasizer ${ }^{\circledR}$ Nano ZS (Malvern Panalytical, Worcestershire, UK). This quasi-elastic light-scattering instrument is equipped with a 4mW Helium-Neon laser, with an output wavelength of $633 \mathrm{~nm}$ and a scatter angle fixed at $173^{\circ}$. The curve fitting of the correlation functions was performed using an exponential fit (Cumulant approach) for the Z-ave and PdI determinations. The Smoluchowski approximation was used to determine the electrophoretic mobility required for $\mathrm{ZP}$ determination. All measurements were performed in triplicate at $25^{\circ} \mathrm{C}$ on LNC suspensions at a concentration of $5 \mathrm{mg} / \mathrm{mL}$ (diluted in deionized water).

\section{Incubation of the NFL Peptide and LNC Suspension}

After their characterization, $1.1 \mathrm{~mL}$ of LNGs at various concentrations in the suspensions, from 0.003 to $300 \mathrm{mg} / \mathrm{mL}$ (diluted in deionized water), was incubated overnight at room temperature with $0.1 \mathrm{~mL}$ of NFL peptide solubilized in deionized water at various concentrations, from 0.6 to $2.4 \mathrm{mg} / \mathrm{mL}$.

\section{Separation Using a Centrifugal-Filtration Device}

A volume of $500 \mu \mathrm{L}$ of LNC suspensions at various concentrations (from 10 to $300 \mathrm{mg} / \mathrm{mL}$ ) or NFL peptide $(0.1 \mathrm{mg}$ / $\mathrm{mL}$ ), as well as LNC/NFL peptide mixtures after incubation, were centrifuged through $100 \mathrm{~K}$ Amicon ${ }^{\circledR}$ Ultra filters for $30 \mathrm{~min}$ at $4000 \times \mathrm{g}$. The filtrate volume was measured by pipetting. The NFL peptide concentration in the filtrate was analyzed using the bicinchoninic acid (BCA) assay or the SEC/UPLC technique (sections below). The presence of LNCs in the filtrate was characterized by assessing their size distribution (with adequate dilution in deionized water) or by Transmission Electron Microscopy (TEM).

\section{Transmission Electron Microscopy}

TEM-negative staining electron microscopy was performed at the Microscopy Rennes Imaging Center platform (MRic TEM) (Univ Rennes, CNRS, Inserm, BIOSIT - UMS 3480, US_S 018, F-35000 Rennes, France). Four-microliter samples were deposited onto glow-discharged electron microscope grids for $1 \mathrm{~min}$ and negatively stained with $2 \%$ uranyl acetate for $10 \mathrm{~s}$. The samples were observed using a $200 \mathrm{kV}$ electron microscope (Tecnai $\mathrm{G}^{2}$ T20 Sphera, FEI) equipped with a $4 \mathrm{k} \times 4 \mathrm{k}$ CCD camera (model USC 4000, Gatan Inc.). Micrographs were acquired using the camera in binning mode 1 and at a nominal magnification of 50,000 $\times$, providing a pixel size of $0.22 \mathrm{~nm}$.

\section{Quantification of NFL Peptide Concentration Using the BCA Assay}

Seven standards (25 to $175 \mu \mathrm{g} / \mathrm{mL})$ and one blank $(0 \mu \mathrm{g} / \mathrm{mL})$ were prepared from the NFL peptide in deionized water. The supplier recommendations were observed. The reagent was prepared by mixing 50 parts BCA reagent $\mathrm{A}$ and 1 part BCA reagent $\mathrm{B}$ (50:1 ratio, $\mathrm{A}: \mathrm{B})$. Each standard and NFL samples $(25 \mu \mathrm{L})$ were added in triplicate to microplate wells, followed by the addition of $200 \mu \mathrm{L}$ reagent. The microplate was mixed for $30 \mathrm{~s}$, covered, and incubated at $37^{\circ} \mathrm{C}$ for $30 \mathrm{~min}$. After cooling the plate, the absorbance was measured at $562 \mathrm{~nm}$ on a SpectraMax ${ }^{\circledR}$ M2 multi-mode microplate reader (Molecular Devices, San Jose, CA, USA). The NFL peptide concentration in the various samples was determined before and after separation $\left(100 \mathrm{~K}\right.$ Amicon ${ }^{\circledR}$ Ultra filters), using the NFL peptide calibration curve (coefficient of determination: $\left.\mathrm{R}^{2}=0.9940\right)$.

\section{Quantification of NFL Peptide Concentration Using the Size-Exclusion Chromatography / Ultra-Performance Liquid Chromatography System}

The free NFL peptide concentrations in the samples (without or with LNGs after the incubation) were directly measured using an Acquity ${ }^{\circledR}$ H-Class Bio UPLC apparatus (Waters, Saint-Quentin-en-Yvelines, France). Briefly, the LNG suspensions, the NFL peptide in solution as well as LNC/NFL peptide mixtures after incubation, at various concentrations, were filtered through $0.22 \mu \mathrm{m}$. The separation between the LNCs and free NFL was carried out using an Acquity UPLC Protein BEH SEC column (200 A, $1.7 \mu \mathrm{m}, 4.6 \mathrm{~mm} \times 300 \mathrm{~mm})$, with an Acquity UPLC@ Protein BEH SEG Guard Column $(200 \AA, 1.7 \mu \mathrm{m}, 4.6 \mathrm{~mm} \times 30 \mathrm{~mm}, 10 \mathrm{~K}-500 \mathrm{~K})$, at room temperature under isocratic conditions (mobile phase: $\mathrm{NaCl}$ in water at $0.1 \mathrm{M}$ ). The flow rate was maintained at $0.3 \mathrm{~mL} /$ min, the injection volume was $10 \mu \mathrm{L}$, and the detection wavelength of the UV detector set to $220 \mathrm{~nm}$. Free peptide 
concentrations were quantified using the area under the peak compared to the concentration of known NFL peptide standards. The calibration curves were freshly prepared and reinjected at the beginning of each analysis.

\section{Statistical Analysis}

Statistical evaluation of all the data was performed using the non-parametric analyses, such as Kruskal-Wallis test followed by Nemenyi-Dunn's post hoc test for pairwise comparisons, or Mann-Whitney test. Only the statistical evaluation of both NFL concentrations before and after separation using centrifugal-filtration device was done thanks to the nonparametric Wilcoxon test, as the samples are paired. The difference between the groups was accepted as significant with $p$ values lower than 0.05 .

\section{RESULTS AND DISCUSSION}

\section{A Size-Exclusion Chromatography System as a Promising Tool}

Numerous studies have reported the use of centrifugalfiltration devices to separate nanosystems from active agents (peptides, xenobiotics, etc.) to quantify their adsorption or encapsulation rates. This is particularly true for LNGs (12,23-28), and the studies of LNG/NFL peptide association $(13,20,24)$. Generally, after separation using the devices, the BCA assay or liquid chromatography is used to quantify the proportion of free NFL peptide in the filtrate and the proportion of adsorbed NFL peptide at the LNG surface is then evaluated. Regarding this technique, two steps are needed to quantify the proportion of free NFL: separation of the free NFL from that adsorbed to the LNC surface and its quantification using the BCA assay or another technique.

We decided to combine separation and quantification in one single step. Based on the literature, free molecules should be separable from nanoparticles using Size-Exclusion Chromatography (29-32). Yang et al. used Sephadex ${ }^{\circledR}$ columns to separate free plasmid DNA from liposomes (33). This type of column was also used to isolate nonencapsulated naringenin from solid lipid nanoparticles (34), and to isolate fluorescently-labelled LNCs in suspension (35). However, this technique is time consuming, requires much solvent, and no quantification of the proportion of free molecule can be performed during the separation. A chromatographic apparatus using specific SEC columns: Acquity UPLC Protein BEH SEC column $(200 \AA$, $1.7 \mu \mathrm{m}, 4.6 \mathrm{~mm} \times$ $300 \mathrm{~mm}$ ), allows the separation of protein mixtures. Their concentrations can be determined at the same time as the separation process (based on the difference in molecular weights) using appropriate detectors and calibration curves specific for the analyzed materials. This concept was transposed to a mix of nanoparticles and peptides, through a case study of LNCs and NFL peptide, and this is the first application of such a technique for nanoparticles to our knowledge, using an UPLC apparatus.

LNCs with a Z-ave of $50 \mathrm{~nm}$ and a $\mathrm{PdI}<0.1$ were formulated according to a phase-inversion method $(21,22)$, with a Span composition of $20 \%\left(w / w_{L N C}\right)$ and no surface charge (Supplementary Material Table 2). First, we injected the LNC suspensions at various concentrations (from 0.003 to $300 \mathrm{mg} / \mathrm{mL}$ ) into the column, which were detected with a retention time of 3.4 min (Fig. 1a-i). The method for LNC quantification using this technique was validated according to ICH Q2 (R1) standards (36). The linearity of the method was shown with LNC concentrations from 1 to $30 \mathrm{mg} / \mathrm{mL}$, repeated 3 times on 3 different days, with coefficient of determination for the mean standard curve of $\mathrm{R}^{2}=0.9992$. The accuracy and the repeatability of the method were assessed and the LNC concentration values to determine were highly lower than $5 \%$ (recommended percentage for method validation) from the accepted true concentration values. The limits of detection and quantification were established at 0.003 and $0.1 \mathrm{mg} / \mathrm{mL}$, respectively.

Secondly, we injected the NFL peptide solution at various concentrations (from 0.01 to $0.5 \mathrm{mg} / \mathrm{mL}$ ) into the column and all were detected after $7.7 \mathrm{~min}$ (Fig. la-iii). The linearity of the method was shown with NFL concentrations from 10 to $150 \mathrm{mg} / \mathrm{mL}$, repeated 3 times on 3 different days, with coefficient of determination for the mean standard curve of $\mathrm{R}^{2}=$ 0.9978. The accuracy and the repeatability of the method were assessed and the NFL concentration values to determine were highly lower than 5\% from the accepted true concentration value. The limits of detection and quantification were established at about 0.0001 and $0.001 \mathrm{mg} / \mathrm{mL}$, respectively.

Finally, we tested the SEC/UPLC technique with a mixture of LNCs and NFL peptide after incubation (overnight at room temperature) and two distinct peaks were detected (Fig. 1a-ii), each corresponding to the previous fraction detected independently. This method can thus be used to separate and directly quantify the proportion of free NFL relative to that adsorbed onto the LNCs. In the absence of prior separation of the incubated LNG/NFL peptide mixtures (no centrifugal-filtration step), quantification by SEC/UPLC showed the proportion of free NFL peptide to be approximately $35 \%$ and $0 \%$ when the LNC suspension concentrations were 1 and $30 \mathrm{mg} / \mathrm{mL}$, respectively (initial NFL concentration of $0.1 \mathrm{mg} / \mathrm{mL}$ ) (Fig. 1b).

We compared the SEG/UPLG technique with the previous one routinely used to study LNC/NFL peptide mixtures with the protocol described in literature for this case study, i.e. a separation step using centrifugal-filtration devices $(100 \mathrm{~K}$ Amicon ${ }^{\circledR}$ Ultra filters for $30 \mathrm{~min}$ at $4000 \times \mathrm{g}$ ) followed by the quantification step using BCA assay to determine the 

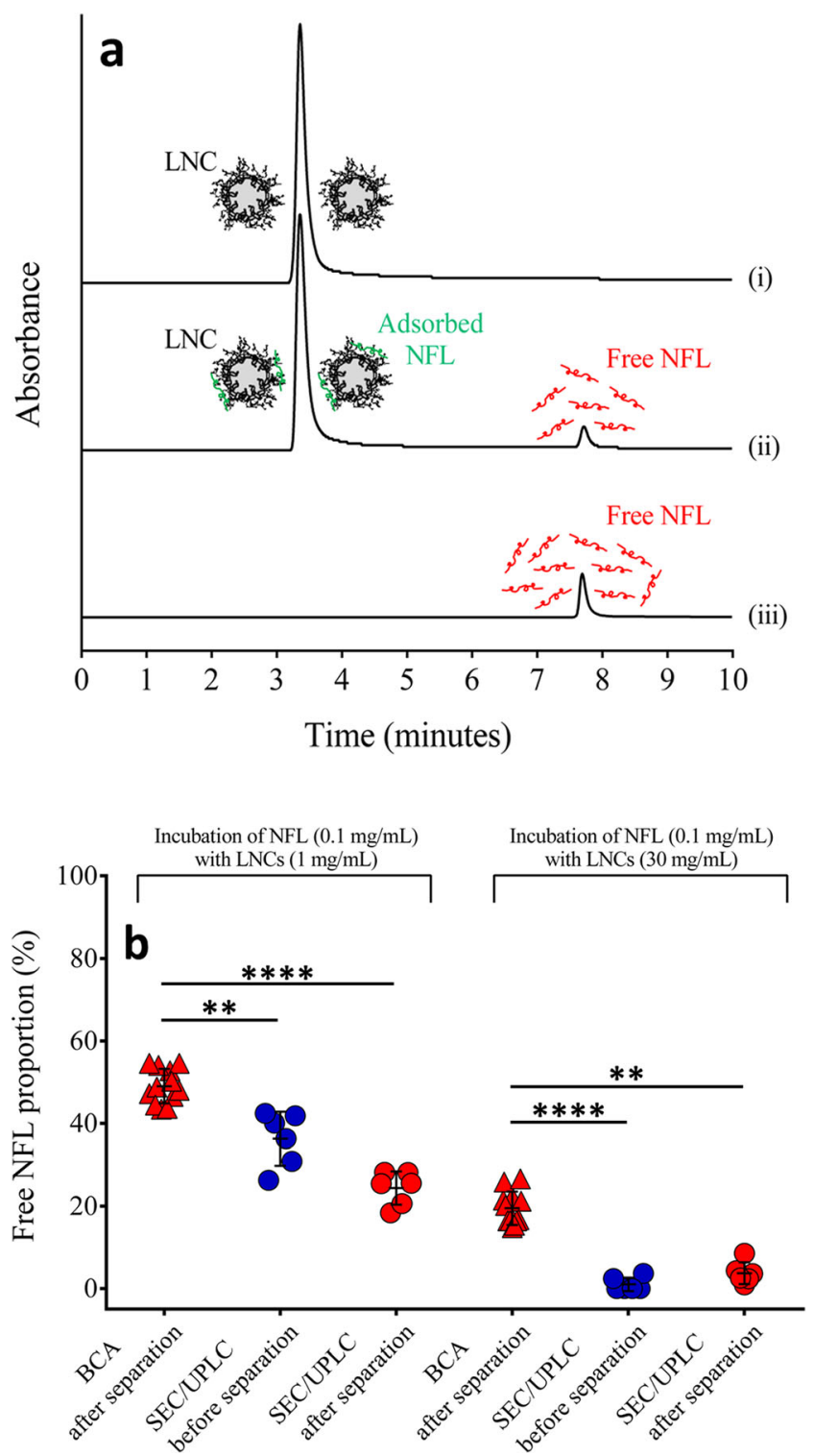

Fig. I Characterization of NFL peptide adsorption to the surface of LNCs without a prior separation step is possible with SEC coupled with UPLC (a) Chromatograms of (i) LNC suspension $(10 \mathrm{mg} / \mathrm{mL}$ ), (ii) a mixture of LNC suspension $(10 \mathrm{mg} / \mathrm{mL})$ and NFL peptide $(0.1 \mathrm{mg} / \mathrm{mL})$, incubated overnight at room temperature, and (iii) NFL peptide solution $(0.1 \mathrm{mg} / \mathrm{mL})$. All the chromatograms start from the origin. They have been shifted for better readability. (b) Free NFL proportion measured by the BCA assay or by the SECNPLC technique, before or after the separation using the centrifugal-filtration. The mixtures of LNC suspension ( I or $30 \mathrm{mg} / \mathrm{mL}$ ) and NFL peptide $(0.1 \mathrm{mg} / \mathrm{mL}$ ) were incubated overnight at room temperature before the quantification ( $n=6-9$; mean \pm SD; Kruskal-Wallis and Nemenyi-Dunn's post hoc tests). Centrifugal filtration cut-off: $100 \mathrm{kDa}$; separation protocol for centrifugalfiltration devices: $4000 \times \mathrm{g}$ and $30 \mathrm{~min}$; LNC: Span composition $=0.20$ $\left(\mathrm{W} / \mathrm{w}_{\mathrm{LNC}}\right), Z$-ave $=50 \mathrm{~nm}$ and $\mathrm{Pdl}<0.1$; *** $p<0.01$, ***** $p<0.000 \mathrm{l}$.

proportion of non-adsorbed NFL peptide in the filtrate $(13,20,24)$. After the separation, the quantification using BCA assay showed significantly higher proportions of free NFL peptide: approximately $50 \%(p=0.008)$ and $20 \%$ $(p<0.0001)$ when the LNG suspension concentrations were
1 and $30 \mathrm{mg} / \mathrm{mL}$, respectively. In addition, BCA assay and the SEC/UPLC technique were compared to determine any differences encountered in terms of quantification of the free NFL peptide after the separation using centrifugal-filtration devices. The quantification by SEC/UPLC showed significant lower proportions of free NFL peptide: approximately $25 \%(p<0.0001)$ and $0 \%(p=0.005)$ for the same LNC suspension concentrations, respectively (initial NFL concentration of $0.1 \mathrm{mg} / \mathrm{mL}$ for both quantification methods) (Fig. 1b). In addition, no significant different proportion of free NFL peptide was observed using SEC/UPLC, with or without prior separation using centrifugal devices.

\section{The Limitations of Centrifugal-Filtration Devices}

In comparison to the separation and quantification-combined method previously described, the difference in the proportion of free NFL during the quantification step can be due to the lack of optimization of the prior separation step when using centrifugal-filtration devices. We investigated the limitations of this technique using the LNG suspensions, the NFL peptide solution, as well as the LNC/NFL peptide mixtures, using the same protocols reported in literature for LNCs and NFL peptide $(13,20,24)$. The behavior of the NFL peptide alone (at $0.1 \mathrm{mg} / \mathrm{mL}$ ) was first assessed to determine the selectivity of the centrifugal-filtration devices. Surprisingly, not all NFL peptide was recovered in the filtrate after centrifugation through the filter of the device and a significant loss of approximately $30 \%(p=0.004)$ was observed (Fig. 2a). This result suggests that the peptide may be retained in the dead volume of the filter, even if its molecular weight is largely lower than that of the filter cut-off (2.7 versus $100 \mathrm{kDa}$, respectively). Considering this experiment as a control, the quantification of free NFL peptide in the incubation assays would be imprecise. Using the separation protocol, the initial peptide concentration has to be optimized to be confident to recover the entire NFL peptide in the filtrate. Moreover, the centrifugal speed could be increased to improve the NFL peptide flow. While the centrifugal speed for this centrifugal-filtration devices can be set at 14,000 g, the protocol described in literature recommend a centrifugal speed of $4000 \times \mathrm{g}(13,20,24)$. This limitation could explain the difference observed in term of free NFL concentrations quantified by SEC/UPLG, before and after the separation with centrifugal-filtration device (Fig. 1b).

We also tested various LNC concentrations (from 10 to $300 \mathrm{mg} / \mathrm{mL}$ ) using the device and assessed the presence of LNCs in the filtrate. Surprisingly, LNCs were detected by dynamic light scattering for all LNC concentrations tested (data not shown) and TEM confirmed their presence (Fig. 2b). These results suggest that the LNCs can cross the filter, even if their molecular weights or sizes are much higher than that of the filter cut-off. Thus, centrifugal-filtration devices may not efficiently separate LNGs from a small molecule. 

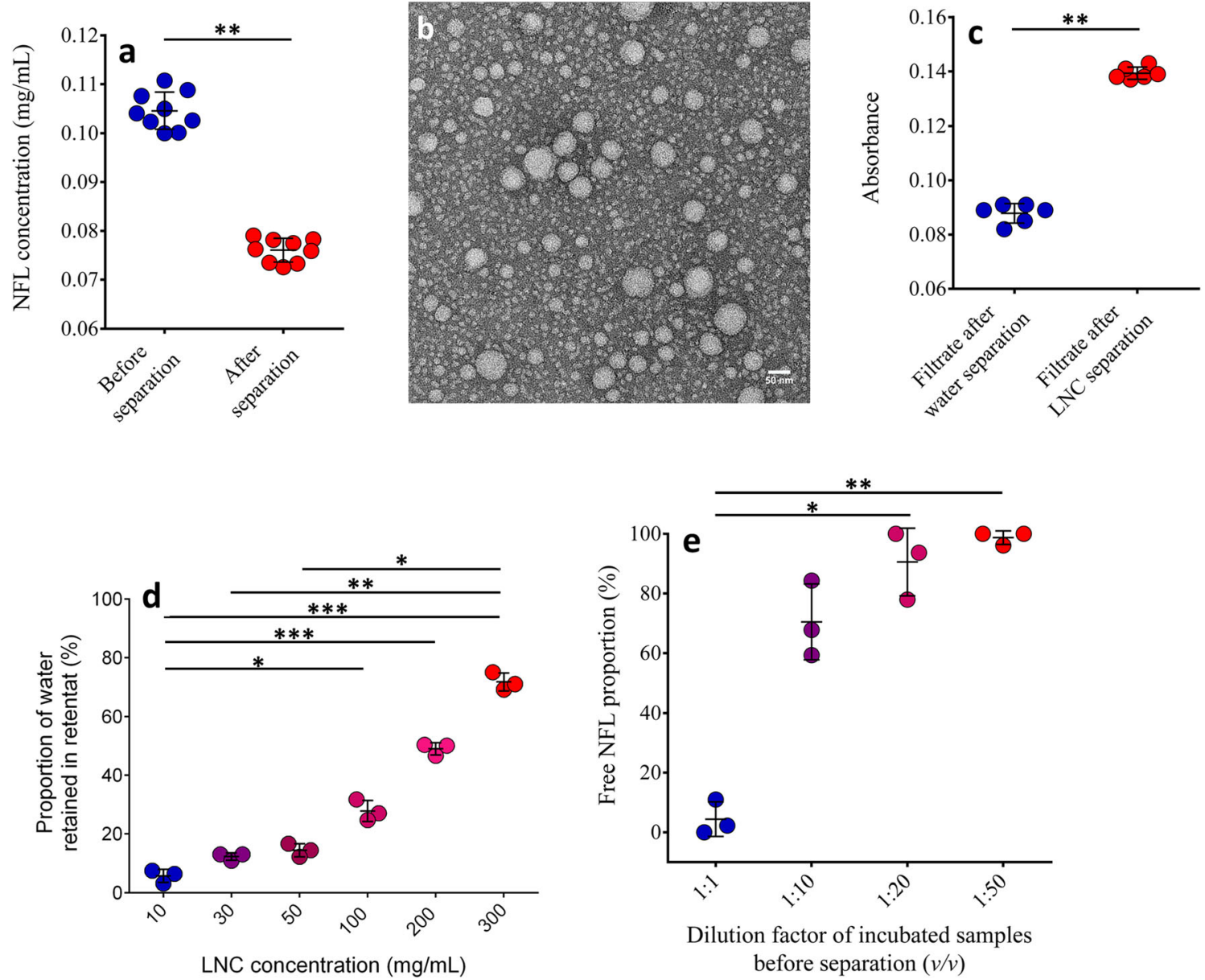

Fig. 2 An accurate quantification of free NFL peptide cannot be evaluated using centrifugal-filtration. (a) NFL peptide concentration measured by the BCA assay, before and after the separation using the centrifugal-filtration ( $n=9$; mean \pm SD; Wilcoxon test). (b) Transmission Electron Microscopy of the filtrate after the separation of LNC suspension $(300 \mathrm{mg} / \mathrm{mL})$ using the centrifugal-filtration. (c) Absorbance of filtrates measured by the BCA assay, after pure water or LNC suspension (300 mg/mL) separation using the centrifugal-filtration ( $n=6$; mean \pm SD; Mann-Whitney test). (d) Proportion of retained water in the retentate after the separation of LNC suspensions (from 10 to $300 \mathrm{mg} / \mathrm{mL}$ ), using the centrifugal-filtration ( $n=3$; mean \pm SD; Kruskal-Wallis and Nemenyi-Dunn's post hoc tests). (e) Free NFL peptide proportions in the filtrate measured by the BCA assay, after the separation using the centrifugal-filtration of a mixture of $L N C / N F L$ peptide (dilution factor with deionized water from I:I to I:50 (v/ )). Before the dilutions, NFL $(800 \mu \mathrm{g} / \mathrm{mL})$ and LNC suspension $(380 \mathrm{mg} / \mathrm{mL})$ were incubated overnight at room temperature $(n=3$; mean $\pm S D$; Kruskal-Wallis and Nemenyi-Dunn's post hoc tests). Centrifugal filtration cut-off: 100 kDa; separation protocol for centrifugal-filtration devices: $4000 \times g$ and $30 \mathrm{~min}$; LNC: Span composition $=0.20$ ( $W / W_{L N C}$ ), Z-ave $=50 \mathrm{~nm}$ and Pdl $<0.1$; * $p<0.05$, *** $p<0.01$, ***** $p<0.001$.

Moreover, a signal in the filtrate was detected by the BCA assay after the filtration of LNCs alone (Fig. 2c), indicating that the presence of LNCs in the filtrate would significantly interfere with the quantification of free peptide or protein using this quantification method $(p=0.005)$. The presence of LNCs in the filtrate has never been reported in previous publications $(12,13,20,23-28)$.

Furthermore, the volume of the aqueous phase contained in the LNC suspensions was not completely recovered after the filtration of the LNCs alone or those incubated with the NFL peptide. We observed a significant loss of volume from 5 to $70 \%$ ( $p=0.001$ ) depending on the LNC concentration (Fig. 2d), suggesting that LNCs may clog the filters and impede the passage of small free molecules, such as the NFL peptide. At the opposite from NFL peptide separation, the increase of centrifugal speed higher than $4000 \times \mathrm{g}$ could worsen the clogging the filter. Finally, we measured the free proportion of NFL peptide after the filtration of various dilutions of incubated LNC/NFL peptide mixtures (concentrations of 380 and $0.8 \mathrm{mg} / \mathrm{mL}$, respectively) to confirm this hypothesis (Fig. 2e). When the incubated sample was not diluted before the separation using centrifugal-filtration device, approximately $5 \%$ of free NFL peptide was recovered in the filtrate, suggesting that almost all of the peptide was adsorbed on the surface of the LNCs. However, when the dilution factor was increased (from 1:10 to 1:50 (v:v)), the proportion of free NFL significantly increased $(p=0.004)$, indicating that the NFL peptide was not adsorbed to the surface of the LNC but retained in the retentate because of the filter clogging due to the LNCs. 
Overall, these results show that centrifugal-filtration devices are not a precise tool to separate LNGs from small molecules to quantify the proportion of free molecule such as NFL peptide, according to the protocol reported in literature $(13,20,24)$. The differences we observed using SEC/UPLG technique and the centrifugal-filtration devices with BCA titration can be due to the combination of all the described limitations, especially the interference due to the presence of LNCs and NFL-adsorbed LNCs in the filtrate. It highlights the need to carefully optimize the characterization methods. Some compromises must be found in terms of centrifugal speed and time, as well as the MW cutoff of the filters, to optimize the separation to reach the right separation between free NFL peptide and NFLadsorbed LNGs. SEC/UPLC allowed the separation and characterization of a mixture of NFL-adsorbed LNGs and free NFL peptide in a single step, thus avoiding the biases mainly due to the separation step before quantification and leading to more precise characterization.
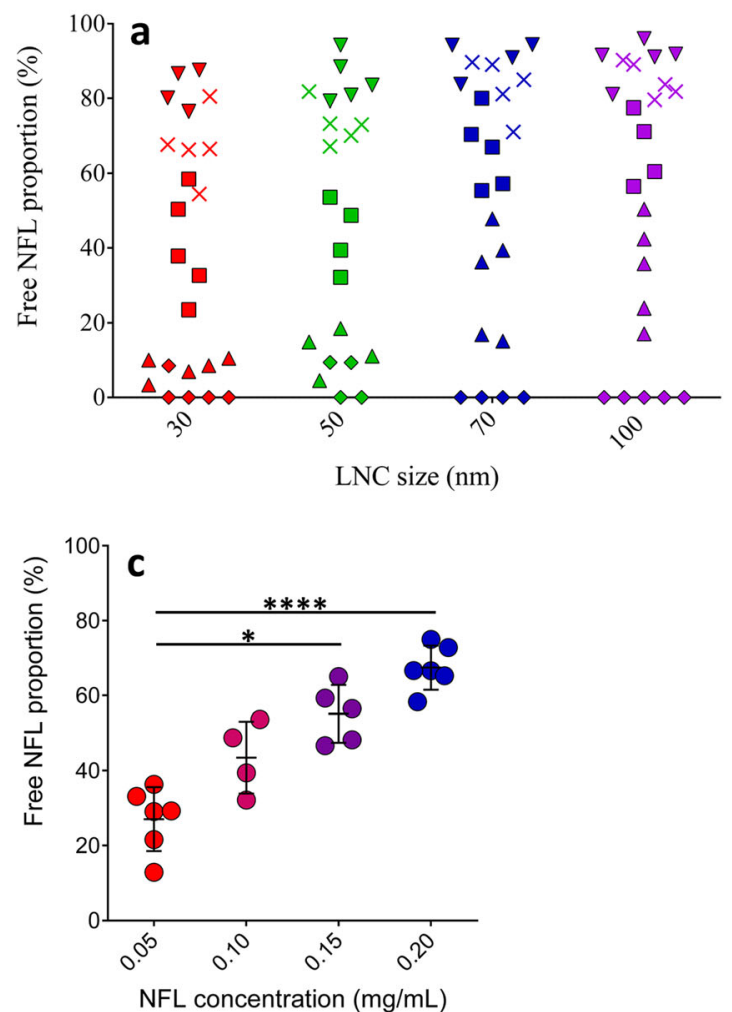

Fig. 3 The NFL adsorption is mediated by the surface of LNCs. Free NFL proportion measured by the SECNPLC technique for the mixtures LNC/NFL peptide, incubated overnight at room temperature, versus (a) the size of LNCs: Z-ave $=30$ (red symbols), 50 (green symbols), 70 (blue symbols) and I00 nm (purple symbols) (Span composition $=0.4 \mathrm{I}$ (w/W surfactant) and Pdl < 0.I); or (b) the total surface area of the LNCs, calculated with the sizes and concentrations of the LNCs in suspension. NFL concentration: $0.1 \mathrm{mg} / \mathrm{mL}$; and LNC concentrations: 100 (diamond), 10 (triangle up), I (square), 0.3 (cross) and $0.1 \mathrm{mg} / \mathrm{mL}$ (triangle down). The grey circles correspond to concentrations of the LNCs in suspension from 0.003 to $300 \mathrm{mg} / \mathrm{mL}$ ( $n=4-5$ ). (c) Free NFL proportion measured by the SEC/NPLC technique for the mixtures LNC/NFL peptide, incubated overnight at room temperature. NFL concentrations: from 0.05 to $0.2 \mathrm{mg} /$ $\mathrm{mL}$; and LNC concentration: I mg/mL (Span composition $=0.2\left(\mathrm{w} / \mathrm{w}_{L \mathrm{NC}}\right)$, Z-ave $=50 \mathrm{~nm}$ and Pdl $\left.<0 . \mathrm{I}\right)(\mathrm{n}=4-5$; mean \pm SD; Kruskal-Wallis and NemenyiDunn's post hoc tests; * $p<0.05$, ****** $p<0.000$ I).

\section{Characterization of the Adsorption of the NFL Peptide to the LNC Surface Using the Size-Exclusion Chromatography System}

The ability to control the amount of adsorbed proteins to nanocarriers is a true benefit because total adsorption removes the need for an additional purification step of the nanoparticles before preclinical in vitro or in vivo experiments. This should speed up the translational procedure to reach the clinical trial phases. The best way to control the total adsorption of NFL peptide to the surface of LNCs is to characterize and understand the adsorption parameters that influence the interaction between the biological and the synthetic materials.

First, we studied the impact of the size of the LNCs. LNCs were designed with a varying but controlled size distribution: Z-ave of 30, 50, 70 and $100 \mathrm{~nm}$, with PdI values lower than 0.1 , implying a monomodal and monodispersed size distribution. The proportion of Span among all the surfactants was kept constant, i.e. $0.41\left(w^{\prime} / w_{\text {Suffactant }}\right)$, to achieve a similar surface composition, independently of the size of the LNCs (Supplementary Material Table 1 and 2 for the

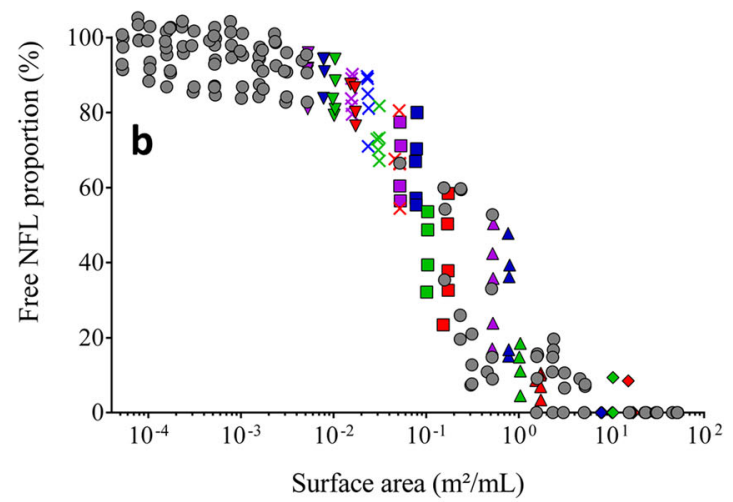



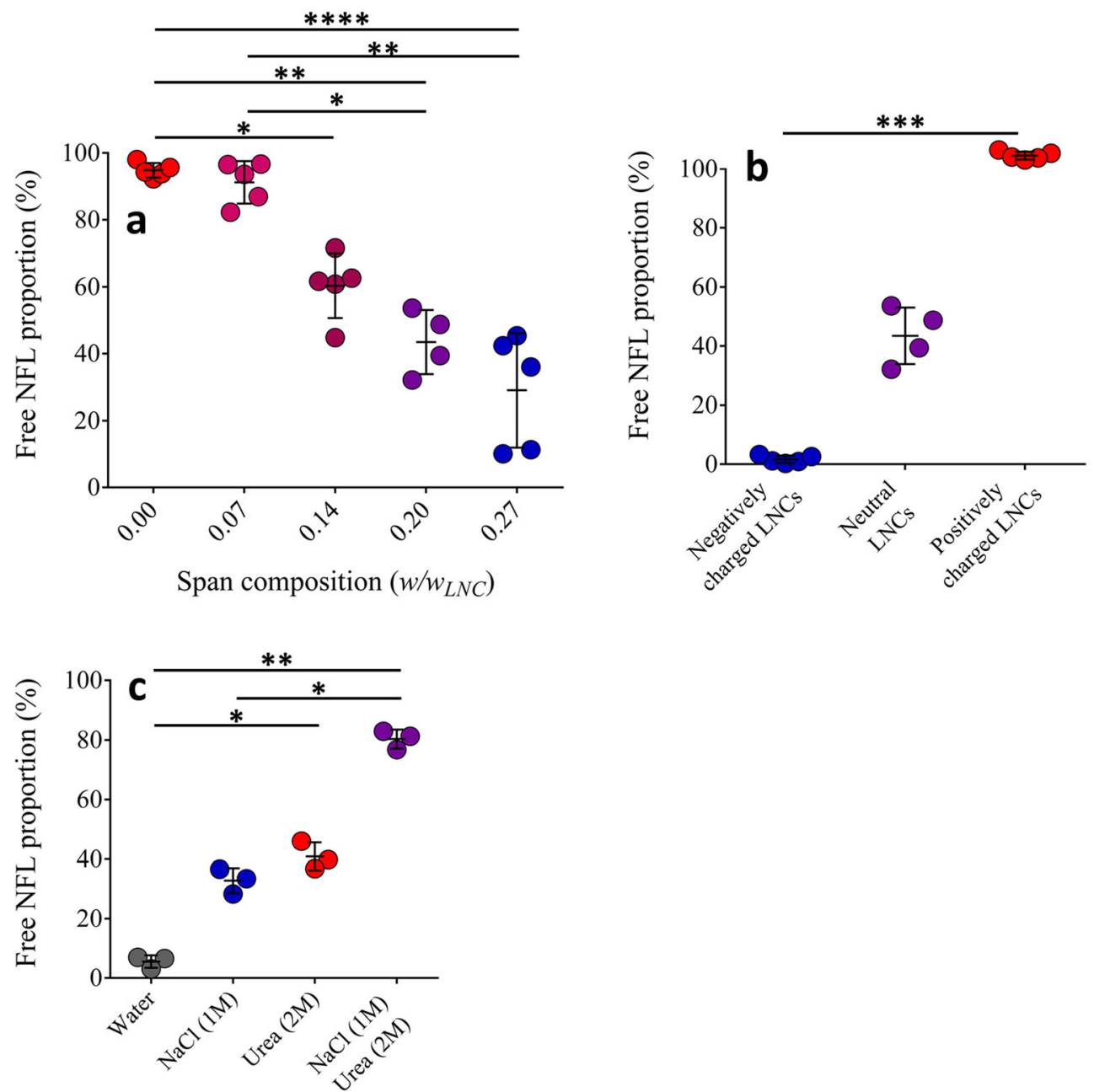

Fig. 4 The NFL adsorption is mediated by the nature of the LNC surface. (a) Free NFL proportion measured by the SEC/NPLC technique for the mixtures LNC/NFL peptide, incubated overnight at room temperature, versus the Span composition in LNCs: from 0 to 0.27 (w/wLNC). NFL concentration: $0.1 \mathrm{mg} / \mathrm{mL}$; and LNC concentration: I mg/mL (Z-ave $=50 \mathrm{~nm}$ and Pdl < 0.I) ( $n=4-5$; mean \pm SD; Kruskal-Wallis and Nemenyi-Dunn's post hoc tests). (b) Free NFL proportion measured by the SEC/NPLC technique for the mixtures LNC/NFL peptide, incubated overnight at room temperature, versus the charge of the surface of the LNCs. NFL concentration: $0.1 \mathrm{mg} / \mathrm{mL}$; and LNC concentration: I mg/mL (Span composition $=0.20\left(w / w_{L N C}\right), Z$-ave $=50 \mathrm{~nm}$ and Pdl $<0.1$ ). Negatively and positively charged LNCs were composed of SChol 0.0125 and DDAB 0.025 (W/WLNC), respectively. Neutral LNCs were neither composed of SChol nor DDAB ( $n=4-5$; mean \pm SD; Kruskal-Wallis and Nemenyi-Dunn's post hoc tests). (c) Free NFL proportion measured by the SEC/NPLC technique for the mixtures LNC/NFL peptide, incubated overnight at room temperature, after the dilution (dilution factor $=1: 4(\mathrm{v}: \mathrm{v})$ ) with deionized water, $\mathrm{NaCl}(\mathrm{I} \mathrm{M})$, urea (2 M), or both. NFL concentration: $0.1 \mathrm{mg} / \mathrm{mL}$; and LNC concentration: $10 \mathrm{mg} / \mathrm{mL}$ (Span composition $=0.20$ ( w/WLNC), SChol composition $=0.0125$ $\left(w / w_{L N C}\right), Z$-ave $=50 \mathrm{~nm}$ and Pdl $\left.<0 . \mathrm{I}\right)(n=3$; mean $\pm \mathrm{SD}$; Kruskal-Wallis and Nemenyi-Dunn's post hoc tests). $* p<0.05, * * * 0.01, * * * *<<0.001, * * * * *$ $p<0.000$ I.

compositions of the formulations and their characterizations, respectively). All of the NFL peptide was adsorbed to the surface of the LNCs for the highest LNC concentrations, regardless of their size. Indeed, no free NFL peptide was observed when $0.1 \mathrm{mg} / \mathrm{mL}$ of the peptide was incubated with LNCs in suspensions at concentrations of $30 \mathrm{mg} / \mathrm{mL}$ (Fig. 1b), $100 \mathrm{mg} /$ $\mathrm{mL}$ (Fig. 3a), and higher (Fig. 3b). At lower concentrations of LNCs, there were fewer LNCs available to adsorb the NFL peptide, regardless of their size, shown by higher proportions of free peptide in the mixture (Fig. 3a). We used the proportion of free NFL peptide versus the total surface area of the LNCs to generate a model curve (Fig. 3b). Total adsorption of
NFL peptide to the surface of the LNCs can be considered for a total surface area of approximately $15 \mathrm{~m}^{2} / \mathrm{mL}$ for the LNCs in suspension, regardless of their size. Thus, we verified the adsorption process mediated by the total available surface of the nanocarriers. We then performed the reverse experiment, keeping the LNG concentration constant: $1 \mathrm{mg} / \mathrm{mL}$ (Span $0.41\left(w / w_{\text {Surfactant }}\right)$, Z-ave $=50 \mathrm{~nm}$ and $\left.\mathrm{PdI}<0.1\right)$, while changing the NFL peptide concentrations from 0.05 to $0.2 \mathrm{mg} / \mathrm{mL}$. Overnight incubation, at room temperature, with increasing amounts of NFL peptide led to a significant increasing proportion of free NFL peptide, from approximately 25 to $65 \%(p<0.0001)$ (Fig. 3c). We thus confirmed 
a surface process for the adsorption of NFL peptide to the surface of LNCs, with increasing amounts of free NFL peptide when the LNC surface was saturated.

We modified the LNG surface by increasing the proportion of Span to improve adsorption of the NFL peptide. We also modified the LNG surface using SChol or DDAB to generate LNCs with a negatively or positively charged surface, respectively (Supplementary Material Table 1 and 2 for the compositions of the formulations and their characterizations, respectively). The proportion of free NFL significantly decreased from approximately 95 to $30 \%(\mathrm{p}<0.0001)$ when the amount of Span in the LNCs was increased from 0 to $0.27\left(w / w_{L N C}\right)$ (Fig. 4a). Giving the surface of the LNGs a negative charge (SChol at $0.0125\left(w / w_{L N C}\right)$ ) led to complete adsorption of the NFL peptide, whereas giving them a positive charge (DDAB at $0.025\left(w / w_{L N C}\right)$ ) completely abolished adsorption of the NFL peptide (Fig. 4b). A neutral LNC surface (without either SChol or DDAB) led to partial adsorption of the NFL peptide to the surface of the LNCs of approximately 45\%. Concerning biological materials, especially peptides, H-bonds are largely reported in the literature to be involved in their interaction with substrates $(37,38)$, as well as with synthetic materials, such as adsorption to the surface of nanoparticles $(39,40)$. Thus, the interaction between NFL and the sorbitan functions of Span at the surface of LNCs through H-bonds is not surprising. In addition, the NFL peptide has been reported to be slightly positively charged, with two arginine residues in its peptide sequence $(17,41)$. Thus, the involvement of electrostatic interactions is likely, attractive for the negatively charged LNGs and repulsive for those that are positively charged.

We verified that improved adsorption was governed by $\mathrm{H}$-bonds and electrostatic interactions by incubating LNCs to which the NFL peptide was completely adsorbed (obtained after a one-night incubation at room temperature of $10 \mathrm{mg} / \mathrm{mL}$ LNCs (Span 0.2 and SChol 0.0125 $\left(w / w_{L N C}\right), Z$-ave $=50 \mathrm{~nm}$ and $\left.\mathrm{PdI}<0.1\right)$ in suspension and $0.1 \mathrm{mg} / \mathrm{mL}$ NFL peptide) with $\mathrm{NaCl}$, urea, or both. The addition of $\mathrm{NaCl}(1 \mathrm{M})$ or urea (2 M) led to the desorption of the NFL peptide. Free NFL peptide was recovered, with proportions of approximately 30 and $40 \%$, respectively, showing that electrostatic interactions (inhibited by the addition of $\mathrm{NaCl}$ ) and $\mathrm{H}$-bonds (inhibited by the addition of urea) are the main forces involved in the adsorption of the NFL peptide to the surface of LNCs. We verified the complementarity of the two forces, as the addition of both $1 \mathrm{M} \mathrm{NaCl}$ and $2 \mathrm{M}$ urea led to a significant higher desorption, resulting in $80 \%$ free NFL peptide $(p=0.002)$, determined by the SEC/ UPLC technique (Fig. 4c).

\section{CONCLUSION}

Precise characterization of the adsorption of biological material onto nanocarriers is crucial for the accuracy of preclinical results, such as in vitro nanocarrier-cell interactions, in vivo nanocarrier journey, etc. Such preclinical studies are important because they are the essential steps before clinical studies. Promising but inaccurate results due to poor characterization can lead to the failure of the translational steps required to obtain long-awaited medicines. Our case study of the adsorption of the NFL peptide to the surface of LNCs highlights that the classical and increasing used approach such as centrifugalfiltration device must be challenged. We established the limit of the separation between free NFL peptide and NFLadsorbed LNCs, which can lead to mis-quantification if the protocol for separation is not carefully optimized. We demonstrated that the characterization of peptide adsorption to a lipid nanoparticle can be achieved in a single step, combining separation and quantification of the proportion of free peptide. We used a SEC combined with a UPLC system to assess the adsorption of the NFL peptide to LNGs. Furthermore, we studied the adsorption phenomenon and showed it to be mediated through $\mathrm{H}$-bond and electrostatic interactions for the case study involving the NFL peptide and LNGs. This single separation/characterization step truly improves the accuracy and robustness of the data, leading to reproducible results. This simple technique could be a promising tool for the scientific community for characterizing the interaction of other combinations of nanosystems and active biological agents, such as the encapsulation of therapeutic proteins or peptides in liposomes, the study of dynamic of protein corona at the surface of nanoparticles, the characterization of mRNA/lipid nanosystems for vaccine development.

Acknowledgments and Disclosures. This work was carried out within the research program GLIOGEL, financially supported by EuroNanoMed3 (8th call). The authors also thank the « Fondation ARC pour le Recherche sur le Cancer » and the « Ligue Contre le Cancer (Comité du Maine et Loire) » for their financial support. CG and GB designed the experiments. CG, MA, MS, NL and AD conducted the experiments and analyzed the results. CG an GB prepared the figures and wrote the manuscript. PS, JE and GB contributed to methods, funding and/or infrastructures. All authors read and commented the manuscript. The authors declare no conflict of interest.

\section{SUPPLEMENTARY INFORMATION}

The online version contains supplementary material available at https://doi.org/10.1007/s1 1095-021-03034-8. 


\section{REFERENCES}

1. Joseph SK, Sabitha M, Nair SC. Stimuli-responsive polymeric Nanosystem for Colon specific drug delivery. Adv Pharm Bull. 2020;10(1):1-12.

2. Singh SP, Sirbaiya AK, Mishra A. Bioinspired smart Nanosystems in advanced therapeutic applications. Pharm Nanotechnol. 2019;7(3):246-56.

3. Zhang YM, Liu YH, Liu Y. Cyclodextrin-based multistimuli-responsive Supramolecular assemblies and their biological functions. Adv Mater. 2020;32(3):e1806158.

4. Jiang Q, Zhao S, Liu J, Song L, Wang ZG, Ding B. Rationally designed DNA-based nanocarriers. Adv Drug Deliv Rev. 2019;147:2-21.

5. El-Sawy HS, Al-Abd AM, Ahmed TA, El-Say KM, Torchilin VP. Stimuli-responsive Nano-architecture drug-delivery systems to solid tumor micromilieu: past, present, and future perspectives. ACS Nano. 2018;12(11):10636-64.

6. Houshmand M, Garello F, Circosta P, Stefania R, Aime S, Saglio $\mathrm{G}$, et al. Nanocarriers as magic bullets in the treatment of leukemia. Nanomaterials (Basel). 2020;10(2):276.

7. Zhi D, Yang T, Yang J, Fu S, Zhang S. Targeting strategies for superparamagnetic iron oxide nanoparticles in cancer therapy. Acta Biomater. 2020;102:13-34.

8. Dormont F, Rouquette M, Mahatsekake C, Gobeaux F, Peramo A, Brusini R, et al. Translation of nanomedicines from lab to industrial scale synthesis: the case of squalene-adenosine nanoparticles. J Control Release. 2019;307:302-14.

9. Gioria S, Caputo F, Urbán P, Maguire CM, Bremer-Hoffmann S, Prina-Mello A, et al. Are existing standard methods suitable for the evaluation of nanomedicines: some case studies. Nanomedicine (Lond). 2018;13(5):539-54.

10. de la Calle I, Menta M, Klein M, Séby F. Study of the presence of micro- and nanoparticles in drinks and foods by multiple analytical techniques. Food Chem. 2018;166:133-45.

11. Aznar R, Barahona F, Geiss O, Ponti J, José Luis T, BarreroMorenoJ. Quantification and size characterisation of silver nanoparticles in environmental aqueous samples and consumer products by single particle-ICPMS. Talanta 2017;175:200-208.

12. Aparicio-Blanco J, Sebastián V, BenoitJP, Torres-Suárez AI. Lipid nanocapsules decorated and loaded with cannabidiol as targeted prolonged release carriers for glioma therapy: In vitro screening of critical parameters. Eur J Pharm Biopharm. 2019;134:126-37.

13. Karim R, Lepeltier E, Esnault L, Pigeon P, Lemaire L, LépinouxChambaud C, et al. Enhanced and preferential internalization of lipid nanocapsules into human glioblastoma cells: effect of a surface-functionalizing NFL peptide. Nanoscale. 2018;10(28): $13485-501$.

14. Doran AE, Foran DR. Assessment and mitigation of DNA loss utilizing centrifugal filtration devices. Forensic Sci Int Genet. 2014;13:187-90.

15. Johnsen E, Brandtzaeg OK, Vehus T, Roberg-Larsen H, Bogoeva $\mathrm{V}$, Ademi $\mathrm{O}$, et al. A critical evaluation of Amicon ultra centrifugal filters for separating proteins, drugs and nanoparticles in biosamples. J Pharm Biomed Anal. 2016;120:106-11.

16. Berges R, Balzeau J, Peterson AC, Eyer J. A tubulin binding peptide targets glioma cells disrupting their microtubules, blocking migration, and inducing apoptosis. Mol Ther. 2012;20(7):1367-77.

17. Berges R, Balzeau J, Takahashi M, Prevost C, Eyer J. Structurefunction analysis of the glioma targeting NFL-TBS.40-63 peptide corresponding to the tubulin-binding site on the light neurofilament subunit. PLoS One. 2012;7(11):e49436.

18. Lépinoux-Chambaud C, Eyer J. The NFL-TBS.40-63 antiglioblastoma peptide enters selectively in glioma cells by endocytosis. Int J Pharm. 2013;454(2):738-47.
19. Laine AL, Huynh NT, Clavreul A, Balzeau J, BéjaudJ, Vessieres A, et al. Brain tumour targeting strategies via coated ferrociphenol lipid nanocapsules. Eur J Pharm Biopharm. 2012;81(3):690-3.

20. Balzeau J, Pinier M, Berges R, Saulnier P, Benoit JP, Eyer J. The effect of functionalizing lipid nanocapsules with NFL-TBS.40-63 peptide on their uptake by glioblastoma cells. Biomaterials. 2013;34(13):3381-9.

21. Heurtault B, Saulnier P, Pech B, Proust JE, Richard J, Benoit JP. Lipid nanocapsules, preparation process and use as medicine. $2011 ;$ U.S. Patent 8,057,823 B2.

22. Heurtault B, Saulnier P, Pech B, Proust JE, Benoit JP. A novel phase inversion-based process for the preparation of lipid nanocarriers. Pharm Res. 2002;19(6):875-80.

23. Umerska A, Matougui N, Groo AC, Saulnier P. Understanding the adsorption of salmon calcitonin, antimicrobial peptide AP1 14 and polymyxin B onto lipid nanocapsules. Int J Pharm. 2016;506:191200.

24. Carradori D, Saulnier P, Préat V, des Rieux A, Eyer J. NFL-lipid nanocapsules for brain neural stem cell targeting in vitro and in vivo.J Control Release. 2016;238:253-62.

25. Lollo G, Ullio-Gamboa G, Fuentes E, Matha K, Lautram N, Benoit JP. In vitro anti-cancer activity and pharmacokinetic evaluation of curcumin-loaded lipid nanocapsules. Mater Sci Eng C Mater Biol Appl. 2018;91:859-67.

26. Kim CH, Sung SW, Lee ES, Kang TH, Yoon HY, Goo YT, et al. Sterically stabilized RIPL peptide-conjugated nanostructured lipid carriers: characterization, cellular uptake, cytotoxicity, and biodistribution. Pharmaceutics. 2018;10(4):199.

27. Rozenbaum RT, Su L, Umerska A, Eveillard M, Håkansson J Mahlapuu M, et al. Antimicrobial synergy of monolaurin lipid nanocapsules with adsorbed antimicrobial peptides against Staphylococcus aureus biofilms in vitro is absent in vivo. J Control Release. 2019;293:73-83.

28. Carradori D, Labrak Y, Miron VE, Saulnier P, Eyer J, Préat V, et al. Retinoic acid-loaded NFL-lipid nanocapsules promote oligodendrogenesis in focal white matter lesion. Biomaterials. 2020;230: 119653.

29. Gao D, Tang S, Tong Q. Oleanolic acid liposomes with polyethylene glycol modification: promising antitumor drug delivery. Int J Nanomedicine. 2012;7:3517-26.

30. Zhang H, Zhang FM, Yan SJ. Preparation, in vitro release, and pharmacokinetics in rabbits of lyophilized injection of sorafenib solid lipid nanoparticles. Int J Nanomedicine. 2012;7:2901-10.

31. Bastiat G, Oliger P, Karlsson G, Edwards K, Lafleur M. Development of non-phospholipid liposomes containing a high cholesterol concentration. Langmuir. 2007;23(14):7695-9.

32. Bastiat G, Lafleur M. Phase behavior of palmitic acid/cholesterol/ cholesterol sulfate mixtures and properties of the derived liposomes. J Phys Chem B. 2007;111(37):10929-37.

33. Yang S, Chen J, Zhao D, Han D, Chen X. Comparative study on preparative methods of DC-Chol/DOPE liposomes and formulation optimization by determining encapsulation efficiency. Int $\mathrm{J}$ Pharm. 2012;434(1-2):155-60.

34. Ji P, Yu T, Liu Y, Jiang J, Xu J, Zhao Y, et al. Naringenin-loaded solid lipid nanoparticles: preparation, controlled delivery, cellular uptake, and pulmonary pharmacokinetics. Drug Des Devel Ther. 2016;10:911-25.

35. Simonsson C, Bastiat G, Pitorre M, Klymchenko AS, Béjaud J, Mély $\mathrm{Y}$, et al. Inter-nanocarrier and nanocarrier-to-cell transfer assays demonstrate the risk of an immediate unloading of dye from labeled lipid nanocapsules. Eur J Pharm Biopharm. 2016;98:4756 .

36. International Conference on Harmonization. Validation of analytical procedures: text and methodology (Q2 (R1)) 1995. Available from: https://www.ema.europa.eu/en/documents/scientific- 
guideline/ich-q-2-r 1-validation-analytical-procedures-textmethodology-step-5_en.pdf. Accessed December 20, 2020.

37. Caporale A, Martin AD, Capasso D, Focà G, Sandomenico A, D'Andrea LD, et al. Short PIGF-derived peptides bind VEGFR-1 and VEGFR-2 in vitro and on the surface of endothelial cells. J Pept Sci. 2019;25(5):e3146.

38. Han J, Ji Y, Youn K, Lim G, Lee J, Kim DH, et al. Baicalein as a potential inhibitor against BACE1 and AChE: mechanistic comprehension through In Vitro and computational approaches. Nutrients. 2019;11(11):2694.

39. Grasso G, Mercuri S, Danani A, Deriu MA. Biofunctionalization of silica nanoparticles with cell-penetrating peptides: adsorption mechanism and binding energy estimation. J Phys Chem B. 2019;123(50):10622-30.

40. Aliakbar Tehrani Z, Jamshidi Z, Jebeli Javan M, Fattahi A. Interactions of glutathione tripeptide with gold cluster: influence of intramolecular hydrogen bond on complexation behavior. J Phys Chem A. 2012;116(17):4338-47.

41. Bocquet A, Berges R, Frank R, Robert P, Peterson AC, Eyer J. Neurofilaments bind tubulin and modulate its polymerization. $\mathrm{J}$ Neurosci. 2009;29(35):1 1043-54.

Publisher's Note Springer Nature remains neutral with regard to jurisdictional claims in published maps and institutional affliations. 